\title{
REGISTRO DE PINUS DISCOLOR BAILEY ET HAWKSWORTH EN LA SIERRA DE MONTE GRANDE, SAN LUIS POTOSI, MEXICO
}

\author{
Jose Antonio Avila Naranjo \\ Edmundo Garcia Moya \\ Juan Antonio Reyes Aguero \\ Centro de Botánica \\ Colegio de Postgraduados \\ 56230 Chapingo, Edo. de México
}

\section{RESUMEN}

Se confirma la presencia de Pinus discolor en una serranía aislada en la región centro norte del estado de San Luis Potosi. El análisis de la posición de los estomas en ambas caras de las acículas, el número de acículas por fascículo, de su longitud, de la altura y del diámetro basal de los arbustos, así como del tamaño del cono, muestran concordancia con las características de la especie. Sin embargo, se amplia ligeramente la variación conocida al comparar la altura de los arbustos y el número de acículas por fascículo de los individuos de la Sierra de Monte Grande con los valores correspondientes observados en otras poblaciones de la misma especie.

\section{ABSTRACT}

The presence of a Pinus discolor forest in an isolated sierra of north central San Luis Potosi state has been confirmed. Position of stomata on both leaf surfaces, leaf length, number of leaves per fascicle, height, basal diameter of the shrubs and cone size correpond to the characteristics of this species. On the other hand, plant height and number of leaves per fascicle increased slightly the known species variation in other pinyon forests.

La exploraciones botánicas realizadas durante 1989 y 1990 en la Sierra de Monte Grande, al norte del municipio de Charcas, San Luis PotosI, permitieron localizar una población de Pinus discolor, que previamente se considero como $P$. cembroides por Rzedowski (1965), y que no fue representada cartográficamente por Bailey, Snajberk y Zavarin (1982), ni por Bailey y Hawskworth (1987), en sus mapas de distribución de $P$. cembroides y $P$. discolor. El rodal se ubica a unos $180 \mathrm{~km}$ al norte de otro ya conocido de San Luis Potosí, localizado cerca de La Amapola, en la Sierra de San Miguelito, al suroeste de la capital del estado (Fig. 1).

Esta población cubre una superficie aproximada de 1200 ha y se encuentra en la Sierra de Monte Grande entre $23^{\circ} 17^{\prime}$ y $23^{\circ} 18^{\prime}$ latitud norte y $101^{\circ} 06^{\prime}$ y $10^{\circ} 11^{\prime}$ longitud oeste, en altitudes de 2300 a $2650 \mathrm{~m}$. El sustrato es ígneo de tipo riolítico; predomina el suelo litosol éutrico, aunque en las partes bajas y planas existe el fluvisol calcárico, 


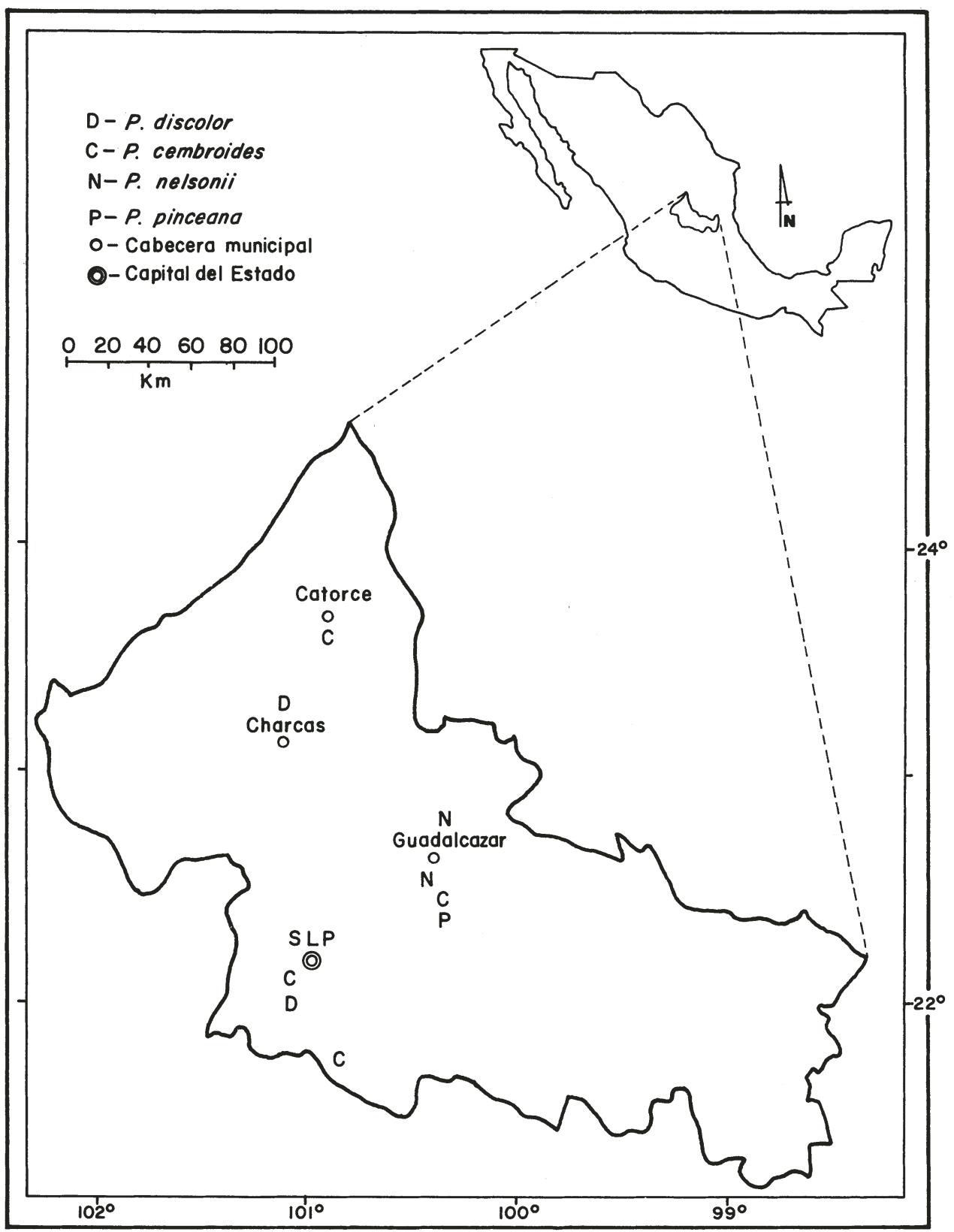

Fig. 1. Distribución de los piñoneros en el estado de San Luis Potosí. (Basado en: Bailey y Hawksworth, 1987; Bailey, Snajberk y Zavarin. 1982). 
su profundidad es menor de $50 \mathrm{~cm}$ (Anónimo, 1971a, b, c). La vegetación del área está constituida por un bosque de pino y encino (Anónimo, 1972).

Durante el verano de 1990 se realizaron recorridos exploratorios por el piñonar; durante dichos recorridos se seleccionaron, al azar, 80 individuos. A cada uno de los arbustos se les constato la presencia o ausencia de estomas en la región abaxial de las acículas; se estimó la altura, diámetro basal, tiempo de retención del fascículo y el sexo.

Los datos registrados en este estudio fueron confrontados con los obtenidos por diversos autores en otras localidades (Cuadro 1). De acuerdo con tal información, los caracteres morfológicos observados en los arbustos de la Sierra de Monte Grande, corresponden a $P$. discolor. Sin embargo, como se puede apreciar en el Cuadro 1, la altura

Cuadro 1. Comparacion de la variables morfológicas de Pinus discolor Bailey. en las diferentes localidades donde se presenta esta especie.

\begin{tabular}{|c|c|c|c|c|c|c|c|c|c|c|c|}
\hline \multirow{2}{*}{ LOC. Y AUTORES } & \multicolumn{2}{|c|}{ ARBUSTOS } & \multicolumn{3}{|c|}{ FASCICULO } & \multicolumn{2}{|c|}{ CONOS } & \multirow{2}{*}{$\begin{array}{l}\text { SEMILLAS } \\
\text { G.T. } \\
(\mathrm{cm})\end{array}$} & \multicolumn{3}{|c|}{ DIOECIA(\%) } \\
\hline & $\begin{array}{l}\text { Alt. } \\
(\mathrm{m})\end{array}$ & $\begin{array}{l}\text { Diám. B. } \\
(\mathrm{cm})\end{array}$ & $\begin{array}{r}\text { Núm. A. Lo } \\
\text { (c }\end{array}$ & m) & $\begin{array}{l}\text { T.R. } \\
\text { (años) }\end{array}$ & $\begin{array}{l}\text { Long. } \\
(\mathrm{cm})\end{array}$ & $\begin{array}{l}\text { Diám. } \\
\text { (cm) }\end{array}$ & & q & $\phi^{\prime}$ & $q^{\prime}$ \\
\hline $\begin{array}{l}\text { Desierto } \\
\text { Chihuahuense }\end{array}$ & & & & & & & & & & & \\
\hline $\begin{array}{l}\text { Bailey y } \\
\text { Hawksworth } \\
(1979,1987)\end{array}$ & $5-12$ & $20-60$ & $\begin{array}{l}3,4,2 \\
\text { y } 5\end{array}$ & $3-6$ & $4-7$ & $2-3$ & $2.5-4$ & $0.5-1.2$ & $>$ & $<$ & - \\
\hline $\begin{array}{l}\text { Zavarin y } \\
\text { Snajberk } \\
(1986)\end{array}$ & 4.9 & - & $2.7-3.8$ & $2.5-4$ & $4 \quad 4-7$ & $2-3$ & $2-2.5$ & $0.8-2.1$ & - & - & - \\
\hline La Amapola, S.L.P. & & & & & & & & & & & \\
\hline Avila (1985) & 4.5 & 17 & 2.99 & 4.3 & - & 2.74 & 3.21 & - & 52.5 & 47.5 & - \\
\hline $\begin{array}{l}\text { Zavala, Avila } \\
\text { y García (1989) }\end{array}$ & 4.7 & 20.03 & 3.05 & 4.49 & 3.8 & - & - & - & 52.0 & 48.0 & - \\
\hline $\begin{array}{l}\text { Avila y García } \\
\text { (1991) }\end{array}$ & 3.53 & 17.5 & 2.96 & 4.4 & - & - & - & - & 46.2 & 43.6 & 10.2 \\
\hline $\begin{array}{l}\text { Sierra del Monte } \\
\text { Grande S.L.P. }\end{array}$ & & & & & & & & & & & \\
\hline Este trabajo & 2.6 & 22.2 & 3.97 & 4.36 & 3.6 & 3.19 & 3.68 & $0.5-2.0$ & 50.6 & 46.2 & 3.2 \\
\hline
\end{tabular}

Abreviaturas y símbolos: Alt., Altura; Diám. B., Diámetro basal; Núm. A., Número de acículas; T.R., Tiempo de retención del fascículo; G.T., Grosor de la testa. > mayor; < menor. 
de los arbustos, el número de acículas por fasciculo y el tiempo de retención del fascículo, manifiestan cierta variación con respecto a lo citado en la literatura para la misma especie.

\section{AGRADECIMIENTOS}

Los autores desean expresar su gratitud a los miembros de comité editorial de Acta Botánica Mexicana, por la revisión crítica que hicieron de este escrito; y por el mismo motivo al Ing. Jorge Pérez de la Rosa.

\section{LITERATURA CITADA}

Anónimo. 1971a. Carta edafológica. F-14-A33. Esc. 1:50 000. Comisión de Estudios del Territorio Nacional. México, D.F.

Anónimo. 1971b. Carta geológica. F-14-A33. Esc. 1: 50 000. Comisión de Estudios del Territorio Nacional, México, D.F.

Anónimo. 1971C. Carta topográfica. F-14-A33. Esc. 1: 50 000. Comisión de Estudios del Territorio Nacional, México, D.F.

Anónimo. 1972. Carta uso del suelo. F-14-A33. Esc. 1: 50 000. Comisión de Estudios del Territorio Nacional, México, D.F.

Avila N., J. A. 1985. Caracterización de los piñoneros (Pinus cembroides Zucc. y $P$. discolor Bailey y Hawks.) de las Serranías Meridionales del estado de San Luis Potosí, México. Tesis Profesional. Escuela de Biologia. Universidad Michoacana de San Nicolás de Hidalgo. Morelia, Michoacán. $101 \mathrm{pp}$.

Avila N., J. A. y E. García M. 1991. Variación morfológica y fenológica de dos especies piñoneras del Altiplano Potosino. Agrociencia, Serie Recursos Naturales Renovables (en prensa).

Bailey, D. K. y F. G. Hawksworth. 1979. Pinyons of the Chihuahuan desert Region. Phytologia 44 (3): 129-133.

Bailey, D. K. y F. G. Hawksworth. 1987. Phytogeography and taxonomy of the pinyon pines (Subsect. Cembroides). In: Passini M. F., D. Cibrián T. y T. Eguiluz P. (comp.). Memorias del II simposio nacional sobre pinos piñoneros. Centre d' Etudes Mexicaines et Centreamercaines, División de Ciencias Forestales (Universidad Autónoma Chapingo), Centro de Genética Forestal A. C. pp. 4164.

Bailey, D. K., K. Snajberk y E. Zavarin. 1982. On the question of natural hybridization between Pinus discolor and Pinus cembroides. Biochemical Systematics and Ecology 10(2): 111-119.

Rzedowski, J. 1965. Vegetacion del estado de San Luis Potosí. Acta Científica Potosina 5(1, 2): 3-291.

Zavala Ch., F., J. A. Avila N. y E. García M. 1989. Variación anual en la longitud de acículas y su relación con los factores ambientales en piñoneros del Altiplano Potosino. In: Flores F., J. D.; J. Flores L.; E. García M. y R. H. Lira S. (comp.). Memorias del III simposio nacional sobre pinos piñoneros. Universidad Autónoma Agraria Antonio Narro, Instituto Nacional de Investigaciones Forestales, Agricolas y Pecuarias. Saltillo, Coahuila. pp. 72-80.

Zavarin, E. y K. Snajberk. 1986. Monoterpenoid differentiation in relation to the morphology of Pinus discolor and Pinus johannis. Biochemical Systematics and Ecology 14(1): 1-11. 